\title{
A double-blind randomized placebo-controlled phase III study of a Pseudomonas aeruginosa flagella vaccine in cystic fibrosis patients
}

\author{
Gerd Döring* ${ }^{\dagger}$, Christoph Meisner ${ }^{\ddagger}$, and Martin Stern ${ }^{\S}$ for the Flagella Vaccine Trial Study Group ${ }^{\Uparrow}$ \\ *Institute of Medical Microbiology and Hygiene, Wilhelmstrasse 31, D-72074 Tübingen, Germany; ${ }^{\ddagger}$ Institute for Medical Information Processing, \\ Westbahnhofstrasse 55, 72074 Tübingen, Germany; and §Children's Hospital, Universitätsklinikum, Hoppe-Seyler-Strasse 1, 72076 Tübingen, Germany
}

Edited by E. Peter Greenberg, University of Washington School of Medicine, Seattle, WA, and approved May 3, 2007 (received for review March 15, 2007)

\begin{abstract}
Pseudomonas aeruginosa causes life-threatening lung infections in patients with cystic fibrosis. We hypothesized that vaccination may prevent $\boldsymbol{P}$. aeruginosa lung infection. In a double-blind, placebocontrolled, multicenter trial, 483 European patients, 2-18 years of age without $P$. aeruginosa colonization were randomly assigned to receive four intramuscular injections of a bivalent $P$. aeruginosa flagella vaccine or placebo over a 14-month period. Patients were evaluated quarterly for $P$. aeruginosa-positive throat cultures and antipseudomonal serum antibody titers during the study period of 2 years. The vaccine was well tolerated, and the patients developed high and long-lasting serum antiflagella IgG titers. In the intentionto-treat group (all patients enrolled), 82 of 239 vaccinated patients had $P$. aeruginosa infection and/or antipseudomonal serum titers compared with 105 of 244 patients in the placebo group $(P=0.05$; relative risk: $0.80 ; 95 \% \mathrm{Cl}: 0.64-1.00)$. Analysis of the 381 patients in the per-protocol group, who received all four vaccinations or placebo treatments, revealed 37 of 189 patients with infection episodes in the vaccine group compared with 59 of 192 patients with such episodes in the placebo group $(P=0.02$; relative risk: $0.66 ; 95 \% \mathrm{Cl}: 0.46-0.93)$. $P$. aeruginosa strains, exhibiting flagella subtypes included in the vaccine, were significantly less frequently isolated from vaccinates than from placebo controls $(P=0.016$, relative risk: $0.319 ; 95 \% \mathrm{Cl}$ : $0.12-0.86)$. Chronic $P$. aeruginosa infection was rare because of recent institution of early antibiotic eradication regimes. Active immunization of patients with cystic fibrosis lowers the risk for infection with $P$. aeruginosa and therefore may contribute to a longer survival of these patients.
\end{abstract}

n patients with cystic fibrosis (CF), life-threatening chronic Pseudomonas aeruginosa lung infections are the leading cause of morbidity and mortality $(1)$, and $\approx 80 \%$ of adult patients with $\mathrm{CF}$ are infected with the pathogen $(2,3)$. The development of mucoid $P$. aeruginosa phenotypes (4) and the high viscosity of mucous plugs prevent the effective killing of the pathogen by neutrophils and antibiotics, leading to chronic infection (5). Consequently, antibiotic-resistant $P$. aeruginosa strains evolve that hamper adequate therapy with these drugs. Although the prevalence of chronic $P$. aeruginosa lung infection in $\mathrm{CF}$ has decreased recently in some countries because of early antibiotic therapy, prevention of $P$. aeruginosa lung infection by immunization may represent a suitable alternative strategy in CF. No vaccine for $P$. aeruginosa infections in $\mathrm{CF}$ patients has yet shown efficacy in a clinical trial (6-8).

We have previously described the safety and immunogenicity of monovalent $P$. aeruginosa flagella vaccines in man $(9,10)$. In healthy human adults, intramuscular immunization resulted in high and long-lasting serum antibody titers against flagella antigens (9). Intramuscular immunization also elicited specific antibodies to flagella of the $\operatorname{IgG}, \operatorname{IgA}$, and secretory IgA Ig isotypes in the secretory immune system of healthy humans (10). $P$. aeruginosa strains that initially colonize $\mathrm{CF}$ patients are generally flagella-positive, composed of "a" and/or " $b$ " flagella subtypes $(11,12)$. Antibodies to $P$. aeruginosa flagella induced by either active or passive immunization are protective in various animal-infection models $(9,13,14)$ and could prevent acute and/or chronic infection in CF patients. Therefore, we immunized CF patients not colonized with $P$. aeruginosa, by using a bivalent $P$. aeruginosa flagella vaccine, containing some of the flagella subtype antigens $\left(\mathrm{a}_{0} \mathrm{a}_{1} \mathrm{a}_{2}\right.$ and $\left.\mathrm{b}\right)$ according to the flagella typing scheme of Ansorg (12), to evaluate its safety and efficacy. We hypothesized that the vaccine would significantly lower the frequency of $P$. aeruginosa infection in $\mathrm{CF}$ patients by at least $66 \%$. This rather low value was chosen on the basis of previous vaccine trials in $\mathrm{CF}$ patients that did not show protection against $P$. aeruginosa infection (6-8).

Author contributions: G.D. and M.S. designed research; G.D. performed research; C.M. analyzed data; and G.D. and M.S. wrote the paper.

The authors declare no conflict of interest.

This article is a PNAS Direct Submission.

Abbreviations: $\mathrm{CF}$, cystic fibrosis; $\mathrm{Cl}$, confidence interval; $\mathrm{FEV}_{1}$, forced expiratory volume in 1 second; ITT, intention-to-treat; PP, per-protocol; PTT, partial thromoblastin time.

${ }^{\dagger}$ To whom correspondence should be addressed at: Institute of Medical Microbiology and Hygiene, Wilhelmstrasse 31, D-72074 Tübingen, Germany. E-mail: gerd.doering@med.unituebingen.de.

"Flagella Vaccine Trial Study Group: S. Brömmea, T. Lietz ${ }^{\mathrm{b}}$, K. Steppberger ${ }^{\mathrm{b}}$, M. Classenc W. Wiebecked, H.-E. Heuere, H.-G. Posselt ${ }^{f}$, G. Dockterg, D. Reinhardth, M. Griese S. Beck' ${ }^{\text {, A. Waagh }}$, S. Beerh, G. Ramakersh, J. Roseneckerh, R. Bertele-Harms', H. K. Harms', K.-D. Pauli, J. Güntherk, G. Krandick', R. J. Franz!, H. Segererm, H. Boehmn, V. Sollicho, A. Schusterp, S. Freudep, M. Schürmann ${ }^{p}$, F. Gudoviusp, H. Lindemannq, P. Bittner-Dersch ${ }^{q}$

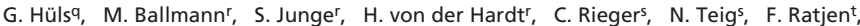
H. V. Bärmeier-Wasmuthu, A. Hebestreity, R. Weisw, W. Rauhw, A. Giuntax, D. Costantinix, L. Marianelliy, G. Taccettiy, G. Bellonz, A. Munckaa, B. Gerardinaa, P. Foucaud bb, C. Ajzen$\mathrm{man}^{\mathrm{bb}}$, I. Eichlercc, S. Rennercc, and R. Rath ${ }^{\mathrm{cc}}$.

aZentrum für Kinderheilkunde, Klinikum Kröllwitz, Martin-Luther-Universität HalleWittenberg, D-06099 Halle, Germany; bKlinik und Poliklinik für Kinder und Jugendliche der Universität Leipzig, D-04317 Leipzig, Germany; CZentralkrankenhaus Links der Weser, Klinik für Kinder- und Jugendmedizin, 28277 Bremen, Germany; dZentralkrankenhaus St.-Jürgen-Strasse, Prof.-Hess-Kinderklinik, 28205 Bremen, Germany; ePraxis Dr. Heuer/Dr. Runge/Dr. Sextro, 22763 Hamburg, Germany; ‘Zentrum für Kinderheilkunde, Klinikum der Johann Wolfgang Goethe-Universität, 60596 Frankfurt, Germany; gUniversitätskinderklinik, 20246 Hamburg, Germany; hKinderpoliklinik der Universität München, 80539 Munich, Germany; 'Dr. von Haunersches Kinderspital der Universität München, 80337 Munich, Germany; JUniversitäts-Kinderklinik, 01307 Dresden, Germany; kPraxis Dr. Günther, 091216 Chemnitz, Germany; 'Kinderklinik Schwabing der Technischen Universität München, 80804 Munich, Germany; ${ }^{m}$ Kinder- und Jugenmedizin der Klinik St. Hedwig, 93049 Regensburg

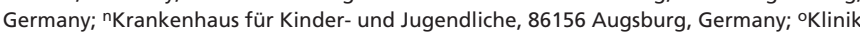
für Kinderheilkunde Otto-von-Guericke-Universität Magdeburg, 39106 Magdeburg, Germany; PZentrum für Kinderheilkunde, Heinrich-Heine-Universität, 40225 Düsseldorf, Germany; 9Zentrum für Kinderheilkunde, Universität Giessen, 35392 Giessen, Germany; rKinderklinik der Medizinische Hochschule Hannover, 30559 Hannover, Germany; ${ }^{5}$ Klinik für Kinder und Jugendmedizin im St. Joseph-Hospital Bochum, Universitätsklinik Bochum, 44787 Bochum, Germany; ${ }^{\text {K}}$ linik und Poliklinik für Kinder- und Jugendmedizin, Universi-

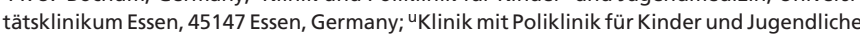
der Friedrich-Alexander-Universität Erlangen-Nürnberg, 91054 Erlangen, Germany; "Kinderklinik und Kinderpoliklinik der Universität Würzburg, 97074 Würzburg, Germany; wKinderabteilung Mutterhaus, Mutterhaus der Borromaerinner, 54290 Trier Germany; ${ }^{\times C l i n i c a}$ Pediatrica II dell'Universita di Milano, 20122 Milan, Italy; YDipartimento di Pediatria "Cesare Cocchi," Ospedale Meyer, 50132 Florence, Italy; zHopital Lyon Sud 69495 Pierre-Bénite, France; aaHopital Robert Debré, 75935 Paris, France; bbCentre Hospitalier de Versailles, Hopital André Mignon, 78157 Le Chesnay Cedex, France; and ccUniversitäts-Kinderklinik, Allgemeines Krankenhaus, A-1010 Vienna, Austria.

This article contains supporting information online at www.pnas.org/cgi/content/full/ 0702403104/DC1.

() 2007 by The National Academy of Sciences of the USA 
Table 1. Characteristics of all subjects with CF enrolled who were randomly assigned to receive the bivalent Pseudomonas flagella vaccine or placebo (ITT) and in fully vaccinated patients (PP)

ITT

PP

\begin{tabular}{|c|c|c|c|c|c|c|}
\hline & & & & & & \\
\hline & Vaccine & Placebo & Total & Vaccine & Placebo & Total \\
\hline Patient numbers & 239 & 244 & 483 & 189 & 192 & 381 \\
\hline Sex (male/female) & $129 / 110$ & $123 / 121$ & $252 / 231$ & $101 / 88$ & 99/93 & $200 / 181$ \\
\hline Age at enrollment, yr & $7.9 \pm 3.9 *$ & $7.0 \pm 4.0$ & $7.5 \pm 3.9$ & $7.8 \pm 4.0$ & $7.1 \pm 4.0$ & $7.4 \pm 4.0$ \\
\hline Weight at enrollment, $\mathrm{kg}$ & $28.2 \pm 13.3^{*}$ & $25.7 \pm 12.9$ & $27.0 \pm 13.1$ & $28.0 \pm 13.6$ & $26.0 \pm 13.3$ & $27.0 \pm 13.5$ \\
\hline $\mathrm{FEV}^{1}(\% \text { of predicted value })^{\dagger}$ at & $98.5(16.1)^{*}$ & $98.7(15.0)$ & $98.6(15.6)$ & $99.8(16.4)$ & $98.7(15.9)$ & $99.3(16.1)$ \\
\hline
\end{tabular}

enrollment ${ }^{\ddagger}$

*Values are means ( \pm values are means SD).

${ }^{\dagger}$ Forced expiratory volume in 1 second.

${ }^{\ddagger} n$ for ITT vaccine, 177; PP vaccine, 140; ITT placebo, 159; PP placebo, 125.

\section{Results}

Assignments of Subjects and Protocol Compliance. The eligibility of 549 patients in $24 \mathrm{CF}$ centers: eighteen in Germany, three in France, two in Italy, and one in Austria was assessed according to inclusion/exclusion criteria in the protocol [supporting information (SI) Fig. 3]. Centers did not differ in their stratification procedure to include patients for the study. Sixty-six patients were excluded because of positive $P$. aeruginosa serum antibody titers as determined by ELISAs in the central laboratory. Two hundred thirty-nine patients were randomly assigned to vaccine and 244 to placebo (Table 1 and SI Fig. 3). Fifty vaccinates were excluded from the per-protocol (PP) analysis because they received fewer than four vaccinations. Forty-six placebo patients were excluded from the PP analysis because they received fewer than four vaccinations, three patients were excluded because the interval between examinations was $>52$ weeks, and another three patients were excluded because both protocol violations were present. Patients with minor protocol violations (age $<2$ years, lung function $<70 \%$; oxygen saturation $<92 \%$, weightto-height $<90 \%$, any missing data) were not excluded for PP analysis to avoid further selection bias. The PP analysis included 189 vaccinates and 193 placebo patients. Patient numbers in vaccine and placebo groups were similar at baseline for both the intention-to-treat (ITT) and the PP analysis and did not differ significantly in sex, age, height, weight, body mass index, and forced expiratory volume in 1 second $\left(\mathrm{FEV}_{1}\right)$ (Table 1$)$. In addition, laboratory values such as bleeding time, partial thromoblastin time (PTT), $\mathrm{CO}_{2}$, and oxygen saturation were not different between groups (data not shown). Reasons for failure to complete the study did not differ between the two groups.

Vaccine Efficacy. With respect to the primary endpoint I (frequency of $P$. aeruginosa infection) there were 82 patients with $P$. aeruginosa-positive throat cultures or positive serum antibody titers to the three $P$. aeruginosa antigens (other than flagella antibodies) or both during the 2-year study period in the vaccine group compared with 105 patients in the placebo group $(P=$ $0.05)$ when the 483 patients in the ITT group were analyzed (Table 2). Analysis of the 381 patients in the PP group, who received all four vaccinations, revealed 37 patients with infection episodes in the vaccine group compared with 59 patients with such episodes in the placebo group $(P=0.02)$. In 18 of the 189 patients in the vaccine group $(9.5 \%)$, positive $P$. aeruginosa throat cultures were not matched by positive antibody titers to $P$. aeruginosa antigens. In the placebo group, 32 of the 192 patients (16.7\%) had positive $P$. aeruginosa throat cultures without positive anti-pseudomonal antibody titers. Descriptively, the PP analysis supports the findings of the ITT analysis.

Although the relative risk in the PP group for having three positive throat swabs and/or three positive serum antibody titers against the three $P$. aeruginosa antigens within a 12-month period was $49 \%$ less in the vaccine group vs. the placebo group (Table 2 ), a statistically significant difference between vaccine and placebo groups was not reached for the primary endpoint II [95\% confidence interval (C.I.): $0.19-1.25]$. There was no difference between the groups concerning the rate of decline in $\mathrm{FEV}_{1}$ during the study period (data not shown).

P. aeruginosa Flagella Typing. To investigate the efficacy of the vaccine to prevent infection of patients' airways with $P$. aeruginosa strains that exhibit flagella subtypes included in the vaccine (flagella subtypes $\mathrm{a}_{0} \mathrm{a}_{1} \mathrm{a}_{2}$ or b), 79 available $P$. aeruginosa isolates from 30 vaccinated patients and 49 placebo patients were typed by indirect immunofluorescence by using two different monoclonal antibodies directed against the flagella subtypes present in the vaccine. Only three strains were flagella-negative, as indicated by the absence of motility in a soft-agar motility assay (data not shown). This indicates that first $P$. aeruginosa infection in $\mathrm{CF}$ patients occurs in the vast majority of cases with flagella-positive strains. Two of the three flagella-negative strains were derived from placebo patients, whereas the third strain was derived from a vaccinated patient.

From the 21 strains reacting with one of the monoclonal antibodies, 16 strains $(76 \%)$ were derived from placebo patients, whereas only 5 strains $(24 \%)$ were derived from vaccinates (Fig. $1)$. The data suggest that vaccination reduced the rate of infection with $P$. aeruginosa strains exhibiting flagella subtypes included in the vaccine (Mantel-Haenszel test, $P=0.016$;

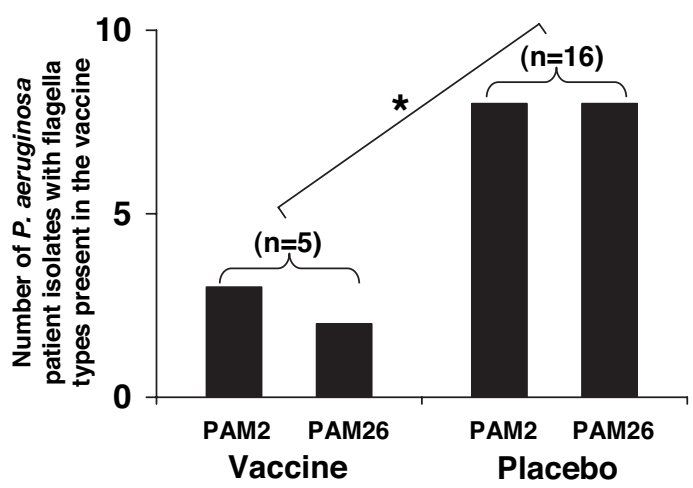

Fig. 1. Vaccination of patients with $\mathrm{CF}$ with the bivalent $P$. aeruginosa flagella vaccine reduces the rate of lung infections with $P$. aeruginosa strains, exhibiting flagella subtypes included in the vaccine. The flagella types present in the vaccine were identified in $P$. aeruginosa throat isolates from CF patients with monoclonal antibodies PAM2 and PAM26, respectively, by using immunofluorescence. * $P=0.016$. 
Table 2. Subjects with CF enrolled (ITT) and fully vaccinated patients (PP) with $P$. aeruginosa lung colonization/infection or chronic infection

\begin{tabular}{lccrcc} 
& \multicolumn{2}{c}{ Primary endpoint I } & & \multicolumn{2}{c}{ Primary endpoint II } \\
\cline { 2 - 3 } Group & \multicolumn{2}{c}{$P$. aeruginosa infection } & & \multicolumn{2}{c}{ Chronic P. aeruginosa infection } \\
\cline { 2 - 3 } & ITT & PP & & ITT & PP \\
\hline Vaccine & $82 / 239 *(34.4)^{\dagger}$ & $37 / 189(19.6)$ & & $26 / 239^{\ddagger}(10.9)$ & $6 / 189(3.2)$ \\
Placebo & $105 / 244(43.0)$ & $59 / 192(30.7)$ & & $29 / 244(11.9)$ & $12 / 192(6.3)$ \\
$P$ & 0.05 & 0.02 & & 0.70 & 0.13 \\
Relative risk & 0.80 & 0.66 & & 0.91 & 0.49 \\
95\% C.I. & $0.64-1.00$ & $0.46-0.93$ & & $0.55-1.49$ & $0.19-1.25$ \\
\hline
\end{tabular}

*Number of patients with minimum of one acute $P$. aeruginosa infection episode during the study period/total patients.

tPercentage.

${ }^{\ddagger}$ Number of patients with chronic $P$. aeruginosa infection/total patients.

relative risk: $0.319 ; 95 \%$ C.I: $0.12-0.86)$. Fifty-eight of the bacterial isolates $(73 \%)$ (isolated from 25 vaccinates and 33 placebo patients) did not react with any of the antibodies directed against flagella subtypes $\mathrm{a}_{0} \mathrm{a}_{1} \mathrm{a}_{2}$ or $\mathrm{b}$, present in the flagella vaccine preparation, although 55 of these strains were motile. This indicated that the majority of patients with positive $P$. aeruginosa isolates had been colonized with strains that exhibited a flagella subtype that was not related to the vaccine antigens.

P. aeruginosa Flagella Antibody Titers. Intramuscular immunization with protein antigens generally leads to high serum antibody titers in vaccinates. When antibody titers against the two flagella antigens present in the vaccine preparation were assessed after the termination of the study, patients in the vaccine group revealed a vigorous immune response to the vaccine antigens Fla1210 and Fla5142 (Fig. 2). Prevaccination, reciprocal serum anti-flagella $\mathrm{IgG}$ titers of all patients from whom serum samples were available $(n=190)$, measured by ELISA, were $<1,500$. Titers increased 4 weeks after the first vaccination to a mean of 7,000 and 7,200 in the vaccine group for Fla1210 and Fla5142, respectively, and remained highly positive during the study period. In contrast, patients in the placebo group had negative anti-flagella IgG titers $<1,000$ when measured at week 20 (Fla $1210, P<0.0001 ;$ median difference vaccine/placebo, 5,940; C.I. 95\%, 5,944-6,001; Fla 5142, $P<0.0001 ;$ median difference vaccine/placebo, 7,958; C.I. 95\%, 791-8,000).

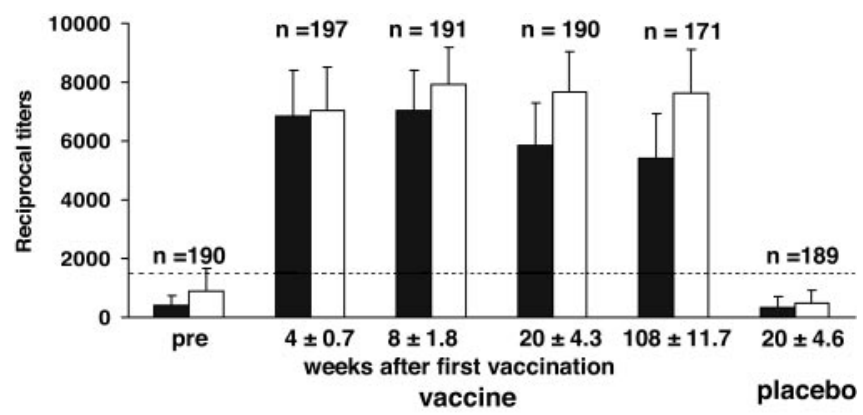

Fig. 2. The bivalent $P$. aeruginosa flagella vaccine elicits high and longlasting specific serum antibody titers against two flagella subtypes in patients with CF. Antibody titers were determined with subtype-specific ELISAs. Filled bars, flagella subtype a0a1a2; open bars, flagella subtype b; n, numbers of patients examined. Time points for the serum collection are given in weeks (mean $\pm \mathrm{SD}$ ). The dotted line represents the cut-off titer, distinguishing negative from positive titers.
Adverse Effects. In general, vaccination was well tolerated by the patients. Less than $5 \%$ of the patients suffered from pressure sensitivity within 1 hour after vaccination (SI Table 3 ). A total of 318 adverse events were registered during the study period within 1 week after vaccination: 227 in the vaccine group and 91 in the placebo group. From these, 312 were classified as moderate or mild, and all patients completely recovered. Five adverse effects were classified as severe (SI Table 4). One event was classified with a definite relationship to the study medication, whereas four adverse effects had no, or an improbable, relationship to the study medication. All but one patient recovered completely from the adverse effect. One adverse effect, not related to the study medication, resulted in death of the patient.

\section{Discussion}

We have demonstrated that active immunization of patients with $\mathrm{CF}$ with the bivalent $P$. aeruginosa flagella vaccine is safe and immunogenic and protects some, but not all, patients against an initial episode of $P$. aeruginosa infection. Local reactions after vaccination were generally mild and resembled those associated with other childhood immunizations. Intramuscular administration of three injections of the vaccine at monthly intervals with a booster 1 year after the third vaccination, led to high concentrations of long-lived flagella-specific serum $\operatorname{IgG}$ antibodies. Based on a previous study (10), it is highly likely that the intramuscular injection of the vaccine also elicited flagellaspecific antibodies of the IgG, IgA, and secretory IgA Ig types in the lung mucosa. A reduction in acute $P$. aeruginosa infection and a reduction the development of antibody titers to $P$. aeruginosa alkaline protease, elastase, and exotoxin A, (primary endpoint I) was observed during the study period in the ITT vaccine group vs. the placebo group, which did not reach significance $(P=0.05 ; 95 \%$ C.I., 0.64-1.00, Table 2). However, this reduction was significant in the 381 patients of the PP group: $30.7 \%$ of patients in the placebo group were colonized with $P$. aeruginosa, whereas, in the vaccine group, only $19.6 \%$ of the patients were colonized $(P=0.02 ; 95 \%$ C.I., $0.46-0.93)$. The PP group was implemented in the study before its start, to exclude patients from the analysis who did not follow precisely the study protocol including quarterly CF center visits and, particularly, those who did not receive all four vaccinations.

Several mechanisms may explain how antibodies to flagella mediate protection from $P$. aeruginosa infection. They may inhibit bacterial adhesion to host cells and molecules, reduce host cell reactions leading to inflammation, block mobility and invasiveness of the pathogen, or induce opsonophagocytosis (13-18). The present findings that acute airway infection of $\mathrm{CF}$ patients with $P$. aeruginosa strains, exhibiting a flagella subtype included in the vaccine preparation, is observed less frequently 
in vaccinates compared with the patients in the placebo group, suggest that opsonophagocytosis and killing of these strains is a major consequence of high antibodies titers to flagella in the vaccine group.

Although we have established an important principle for the prevention of acute $P$. aeruginosa infection in CF patients, the analysis of the flagella types in $P$. aeruginosa isolates by using the monoclonal antibodies PAM2 and PAM26 also suggests that the inclusion of other $P$. aeruginosa flagella types (19) in a future pentavalent vaccine preparation may improve protection against $P$. aeruginosa in $\mathrm{CF}$ patients. This notion is based on the findings that the majority of patients with positive $P$. aeruginosa isolates had been infected with strains that exhibited a flagella type that was not related to the vaccine antigens. A similar finding with regard to the existing heptavalent conjugate vaccine against Streptococcus pneumoniae spurred development of newer vaccines containing 9 or 11 capsular polysaccharide antigens.

Advances in the diagnosis and care of CF patients have not changed the time for initial acquisition of oropharyngeal $P$. aeruginosa. In a recent publication, the median age at first $P$. aeruginosa infection was 1 year (20). However, successful strategies with antibiotic therapy that aim to eradicate $P$. aeruginosa from $\mathrm{CF}$ airways immediately after the detection of the first infection $(21,22)$ have prolonged the transition time to chronic infection. In Wisconsin, CF patients suffer from chronic $P$. aeruginosa infection at a median age of 13 (20), and, in the CF center in Copenhagen, Denmark (not included in the present study), virtually no patient below the age of 15 in the year 2002 was chronically infected, whereas $75 \%$ of comparable CF patients had become chronically infected in the period until 1975 (T. Pressler, personal communication). Although early antibiotic therapy significantly reduced treatment costs (22), compared with therapy for chronically infected patients, the flagella vaccine, given three times initially with boosters every year could be even more cost effective and require less medical intervention, depending on the price of a marketed vaccine preparation and the success rate of the antibiotic treatment strategy in various countries. Ideally, both strategies could be combined. Quarterly microbial cultures and serology to monitor both vaccine efficacy and $P$. aeruginosa status, would allow fine-tuning of booster doses and initiation of inhaled antibiotic therapy.

The recently developed use of antibiotic therapy after detection of $P$. aeruginosa in any throat culture from a CF patient still allows testing the efficacy of a vaccine to prevent acute infection of $\mathrm{CF}$ airways by $P$. aeruginosa, because antibiotics are rarely given prophylactically, and such a treatment is not encouraged by a European consensus statement (5). However, given the apparent ability of this intervention in preventing the development of chronic $P$. aeruginosa infection, it might be difficult to test whether the vaccine prevents chronic $P$. aeruginosa infection. This limitation was encountered in the present study, in which the mean age of the 483 patients was $7.5 \pm 3.9$ years, and the number of chronic $P$. aeruginosa lung infections was much smaller than anticipated before the onset of the study. During the study period, only 2 of 244 patients in the placebo group $(0.82 \%)$ had repeatedly positive $P$. aeruginosa throat cultures and positive antibody titers to $P$. aeruginosa antigens, indicating chronic infection. Therefore, a significant difference between vaccine and placebo group was not observed, although the relative risk in the PP group for suffering from chronic $P$. aeruginosa pulmonary infection was $49 \%$ less in the vaccine group vs. placebo group.

Taken together, this study shows that active immunization of patients with $\mathrm{CF}$ lowers the risk for initial infection with $P$. aeruginosa. Although many of these patients may acquire $P$. aeruginosa infections later in life, delaying the onset of chronic infection with $P$. aeruginosa should result in longer survival of these patients.

\section{Materials and Methods}

Study Subjects. From May 6, 1997, to February, 9, 2000, 483 patients with $\mathrm{CF}$, ranging from 1 to 18 years of age were recruited for the study from 24 European CF centers. Patients were eligible for enrolment if they had $\mathrm{CF}$ that had been diagnosed according to conventional criteria, an age between 2 and 18 years, had no infection with $P$. aeruginosa as assessed by a negative throat swab culture and negative serum antibody titers against the $P$. aeruginosa antigens exotoxin $\mathrm{A}$, and alkaline protease and elastase by using ELISAs wherein a positive titer is defined by the manufacturer (23) (Mediagnost, Reutlingen, Germany). All patients had an initial $\mathrm{FEV}_{1}$ of at least $70 \%$ of the predicted value, a weight-to-height ratio of at least $90 \%$ and an oxygen saturation of at least $92 \%$. Patients were excluded if they had a known allergy to thiomersal or mercury, a prolonged bleeding time or a pathological PTT value, were using immunosuppressive drugs such as systemic corticosteroids, or were participating in other clinical studies. Screening for $P$. aeruginosa infection was carried out 2 weeks before the first vaccination.

Study Design, Randomization, Administration of Vaccine/Placebo, and Safety Study. The phase III study was a randomized, doubleblind, placebo-controlled, multicenter trial. The assumptions for the sample-size calculation were based on the original definition of the primary endpoint and on the assumption that $16 \%$ of patients treated with placebo will be infected by $P$. aeruginosa during the study period of 2 years. Assuming that vaccination would be clinically important in reducing the incidence of $P$. aeruginosa infections by $66 \%, 5.3 \%$ of patients treated with the vaccine should get infected during the study period. A sample size of 160 patients per group was needed for the statistical verification of such a result (Mantel-Haenszel test; one-sided significance level $\alpha=0.05$; power, $90 \%$ ). The choice of the one-sided significance level was based on prior safety data of flagella vaccine preparations in phase I studies. Allowing for drop-outs and withdrawals of up to $20 \%$, recruitment of 400 patients was planned. Informed consent was obtained from all patients or their parents, and the study protocol was approved by the institutional review boards at the participating hospitals, the biostatistician, the International Steering Committee, the $\mathrm{Su}$ pervisory Board, and the respective administrative bodies of the European countries Germany, Italy, France, and Austria. The study was conducted according to International Conference on Harmonisation (ICH)/good clinical practice (GCP) and CONSORT guidelines.

Patients were randomized in blocks of 12 patients in a $1: 1$ ratio between vaccine and placebo by using random numbers, generated by the algorithm of Wichmann and Hill (24), stratified by center. For each patient, a package of four prefilled 1-ml syringes, numbered with the randomization code, and containing either $40 \mu \mathrm{g}$ of flagella protein $(20 \mu \mathrm{g}$ of flagella of subtype $\mathrm{a}_{0} \mathrm{a}_{1} \mathrm{a}_{2}$ from $P$. aeruginosa strain 1210 and $20 \mu \mathrm{g}$ of flagella of subtype b from $P$. aeruginosa strain 5142), $2 \mathrm{mg}$ of aluminum hydroxide, and $0.1 \mathrm{mg}$ of thiomersal or $2 \mathrm{mg}$ of aluminum hydroxide and $0.1 \mathrm{mg}$ of thiomersal only, was provided. Allocation of patients to vaccine or placebo was successfully concealed by the manufacturer who provided the allocation list to the statistician (C.M.) only after closure of data entry at the end of the trial on April 19, 2003. Furthermore, the statistical analysis plan was finalized before unblinding. The patients were assigned by the clinical investigators in the CF centers to the packages in ascending numerical order as they were enrolled consecutively in the study. The patients received the contents of three syringes by intramuscular injection during $\mathrm{CF}$ clinic visits, one syringe every 4 weeks and alternating between the right and left upper arm. 
After 1 year, the content of a fourth syringe was injected in the left upper arm. Patients were physically examined before and 1 hour after the vaccinations for blood pressure, pulse frequency, respiratory rate, and body temperature.

At the beginning of the study, the safety of the vaccine was assessed in $48 \mathrm{CF}$ patients fulfilling the same inclusion criteria as described above. The patients received the first three intramuscular injections of the bivalent vaccine or placebo according to the phase III protocol as described above. One month after the third injection, the number of adverse reactions was compared between vaccine and placebo groups by an independent investigator in a blinded fashion. No statistical difference was noted between the two groups. Based on the decision of a supervisory board, the 48 patients continued the phase III study according to the protocol, and an additional $435 \mathrm{CF}$ patients were enrolled.

Analysis of Adverse Effects and Premature Termination of Study. The following adverse reactions were assessed 1 hour after the injections: pressure sensitivity, redness, swelling, pain, temperature, nausea, chill, headache, limb pain, and any other adverse reactions. Adverse reactions were documented by the patients or their parents at home during the first week after each infection.

Analysis of Outcome Measures. The protocol specified one primary outcome measure for evaluating the efficacy of the vaccine: the lower frequency or complete absence of $P$. aeruginosa infection in the vaccine group compared with the placebo group during the 2-year observation period of the study. Diagnosis of a $P$. aeruginosa infection was defined as having one or more $P$. aeruginosa-positive throat swabs or positive serum antibody titers against the $P$. aeruginosa antigens alkaline proteinase, elastase, and exotoxin A (primary endpoint I). During the study, the protocol was amended, and a primary endpoint II was introduced, based on the identical assumptions as for the original endpoint. The primary endpoint II was defined as three positive throat swabs and/or three positive serum antibody titers against $P$. aeruginosa antigens (see below) within a 12-month period during the study, to assess chronic $P$. aeruginosa infection in the patient groups. The assumptions for the power calculation for the primary endpoint I have also been applied to the primary endpoint II. Secondary criteria for efficacy were $(i)$ a difference between the vaccine and the placebo groups in specific serum antibody titers against the inoculated antigens and (ii) the distribution of $P$. aeruginosa flagella subtype strains between the vaccine and the placebo groups.

Throat swabs were evaluated for $P$. aeruginosa by routine culture in the microbiological laboratories of the CF centers at each visit of the patients. Serum samples taken at each visit and stored at $-20^{\circ} \mathrm{C}$ were assessed by a central laboratory for serum antibody titers to $P$. aeruginosa antigens alkaline proteinase, elastase, and exotoxin A at the former Institute of General and Environmental Hygiene, University of Tübingen. In addition to

1. Ratjen F, Döring G (2003) Lancet 361:681-689.

2. Cystic Fibrosis Foundation (2005) Cystic Fibrosis Foundation Patient Registry 2004 Annual Data Report (Cystic Fibrosis Foundation, Bethesda)

3. Wiedemann B, Steinkamp G, Sens B, Stern M, German Cystic Fibrosis Quality Assurance Group (2001) Eur Respir J 17:1187-1194.

4. Worlitzsch D, Tarran R, Ulrich M, Schwab U, Cekici A, Meyer KC, Birrer P, Bellon G, Berger J, Weiss T, et al. (2002) J Clin Invest 109: 317-325.

5. Döring G, Conway SP, Heijerman HG, Hodson ME, Høiby N, Smyth A, Touw DJ for the Consensus Committee (2000) Eur Respir J 16:749767.

6. Pennington JE, Reynolds HY, Wood RE, Robinson RA, Levine AS (1975) Am J Med 58:629-636.

7. Langford DT, Hiller J (1984) Arch Dis Child 59:1131-1133. the assessment of antibody titers against the three $P$. aeruginosa antigens and to document the immunological reaction to the vaccine antigens, sera were analyzed for antibody titers to the $P$. aeruginosa flagella "a" and flagella " $b$ " subtype antigens by ELISA (10).

The distribution of gender, age, height, weight, and FEV1 (\% of predicted value) were compared in the two groups at the time of admission to the study, by using means and SD, respectively, using frequencies. The statistical evaluation of the primary outcome measures were performed according to the ITT and the PP principle. The null hypothesis of identical incidence rates in the vaccine and placebo groups was tested by using the one-sided Mantel-Haenzel test. The ITT analysis addresses the primary hypothesis of the study. The multicenter nature of the study was taken into account by using stratified methods. The level of significance was set to 0.05 . The results of the statistical tests were given with their nominal $P$ value. For all of the other parameters, the observed effects were described with the usual statistical values such as frequency tables, means, medians, 95\% C.I., and ranges. Data entry was performed by two individuals, independently into an MSAccess 97 database. SAS was used for statistical analysis (version 8.0; SAS Institute, Cary, NC).

$\boldsymbol{P}$. aeruginosa Flagella Typing by Indirect Immunofluorescence. $P$. aeruginosa isolates were cultured overnight in tryptic soy broth (TSB), centrifuged, and washed three times with PBS. Ten microliters of the suspensions were fixed on glass slides with $4 \%$ paraformaldehyde, incubated with swine serum (1:10 in PBS), (Dako, Hamburg, Germany) for $30 \mathrm{~min}$ and then with different monoclonal antibodies against flagella for $1 \mathrm{~h}$ at room temperature. Monoclonal IgG1k antibodies (Baxter, Vienna, Austria), raised against flagella of $P$. aeruginosa strain 1210 (subtype $\mathrm{a}_{0} \mathrm{a}_{1} \mathrm{a}_{2} ;$ PAM2) and strain 5142 (subtype b; PAM26) were used. After washing, antibody binding was detected by using indocarbocanin 3-(Cy3)-conjugated goat anti-mouse IgG (Dako) after incubation in a dark chamber for $1 \mathrm{~h}$. Bacterial DNA was stained with $5 \mu \mathrm{g} / \mathrm{ml}$ DAPI, and the samples were embedded with fluorescence mounting medium (Dako).

We thank Johann Eibl and Friedrich Dorner for the production of the bivalent $P$. aeruginosa flagella vaccine at Immuno AG, Vienna, Austria; Dietrich Niethammer and Bertram Flehmig (Children's Hospital, Universitätsklinikum, Tübingen) for their work in the supervisory board; Dieter Worlitzsch, Kerstin Glück, Anna Smaczny, Maik Häfner, and Maria Haug (Institute of Medical Mikrobiology and Hygiene) for immunological measurements and monitoring; Rainer Stolper and Dagmar Henke (Institute for Medical Information Processing, Universitätsklinikum Tübingen) for data management; and Gerald B. Pier (Harvard Medical School and Brigham and Women's Hospital, Boston, MA) and Brian Crowe (Baxter, Vienna, Austria) for help with manuscript preparation. The study was supported by grants from the Gesellschaft zur Bekämpfung der Mukoviszidose e.V., Bonn, Germany, Vaincre la Mucoviscidose, Paris, France, L'Assoziazione de la Fibrosi Cistica Lombardia, Milan, and Ospedale Meyer, Florence, Italy, and Verband der Cystischen Fibrose, Vienna, Austria.

8. Schaad UB, Lang AB, Wedgwood J, Ruedeberg A, Que JU, Furer E, Cryz SJ, Jr (1991) Lancet 338:1236-1237.

9. Crowe BA, Enzersberger O, Schober-Bendixen S, Mitterer A, Mundt W, Livey I, Pabst H, Kaeser R, Eibl M, Eibl J (1991) Antibiot Chemother 44:143-1456.

10. Döring G, Pfeiffer C, Weber U, Mohr-Pennert A, Dorner F (1995) Am J Respir Crit Care Med 151:983-985.

11. Anderson TR, Montie TC, Murphy MD, McCarthy VP (1989) J Clin Microbiol 62:2789-2793.

12. Ansorg R (1978) Zentralbl Bakteriol Mikrobiol Hyg I Abt Orig A 242:228238.

13. Holder IA, Naglich JG (1986) J Trauma 26:118-122.

14. Landsperger WJ, Kelly-Wintenberg KD, Montie TC, Knight LS, Hansen MB, Huntenburg CC, Schneidkraut MJ (1994) Infect Immun 62:48254830 . 
15. McNamara N, Khong A, McKemy D, et al. (2001) Proc Natl Acad Sci USA 98:9086-9091.

16. Ramphal R, Guay C, Pier GB (1987) Infect Immun 55:600-603.

17. Feldman M, Bryan R, Rajan S, Scheffler L, Brunnert S, Tang H, Prince A (1998) Infect Immun 66:43-51.

18. Adamo R, Sokol S, Soong G, Gomez MI, Prince A (2004 Am J Respir Cell Mol Biol 30:627-634.

19. Spangenberg C, Heuer T, Burger C, Tummler B (1996) FEBS Lett 396:213-217.
20. Li Z, Kosorok MR, Farrell PM, Laxova A, West SE, Green CG, Collins J, Rock MJ, Splaingard ML (2005) J Am Med Assoc 293:581-588.

21. Valerius NH, Koch C, Høiby N (1991) Lancet 338:725-726.

22. Taccetti G, Campana S, Festini F, Mascherini M, Döring G (2005) Eur Respir $J$ 26:1-4.

23. Danielsen L, Westh H, Balselv E, Rosdahl VT, Döring G (1996) Lancet 347:265.

24. Wichmann BA, Hill ID (1982) Appl Stat 1:188-190. 